\title{
Editorial
}

Nephrology

Published online: June 6, 2015

DOI: $10.1159 / 000430924$

\section{Early Kidney Disease, an Important Factor in Cognitive Decline or Merely a Harbinger of Early Vascular Brain Injury?}

\author{
Joshua R. Lewis ${ }^{a} b$ \\ ${ }^{a}$ Centre for Kidney Research, Children's Hospital at Westmead, School of Public Health, Sydney Medical School, The \\ University of Sydney, Sydney, buniversity of Western Australia School of Medicine and Pharmacology, Sir Charles \\ Gairdner Hospital Unit, Perth, Australia
}

Cognitive impairment is common in the elderly and is a major burden on the healthcare system. It is generally accepted that vascular brain injury even in the absence of large vessel disease or stroke is a major underlying cause of cognitive aging; however, the importance of other factors remains uncertain. Cognitive aging is a highly dynamic and potentially modifiable process rather than a specific disease per se. The recent Institute of Medicine report noted that while cognitive aging is difficult to define on a population level, the best indicator is individual performance assessed at several time points [1]. In this regard, an important paper by Seliger and colleagues [2] in this issue of the American Journal of Nephrology reports on the relationship between longitudinal measures of renal and cognitive decline at several time points in the Baltimore Longitudinal Study of Aging. This is a prospective study of 2,116 community-based adults with a mean age of 54 with mild kidney disease and without a history of stroke or dementia. The authors found that declining renal function was associated with impairment of some, but not all cognitive domains using a battery of multidomain cognitive tests, administered by trained psychometricians every 2 years (median of 4 visits per individual). While previous studies have reported that moderateto-end-stage renal disease is an important risk factor for cognitive impairment and the progression of cognitive (c) 2015 S. Karger AG, Basel

0250-8095/15/0415-0303\$39.50/0 decline [3-5] the study by Seliger and colleagues [2] has now extended these findings to people with early kidney disease above the current threshold for clinical concern.

Specifically, in this cohort of men and women with a mean eGFR at baseline of $103 \mathrm{ml} / \mathrm{min} / 1.73 \mathrm{~m}^{2}$, modest increases in creatinine concentrations or decreasing estimated glomerular filtration rate by the MDRD equation were associated with lower function in verbal and visual memory as well as greater rates of decline in these cognitive domains. This relationship was more pronounced in older individuals and in men. Importantly, these relationships remained significant after adjustment for shared vascular risk factors and after the exclusion of individuals with cardiovascular disease and moderate kidney disease. On the other hand, no associations were observed between declining renal function and changes in other cognitive domains such as naming, executive function, language fluency or psychomotor speed supporting the concept that declining renal function affects specific cognitive domains. These findings are in good accord with a longitudinal study of 590 men and women with mild-to-moderate CKD that reported that decline in renal function measured twice in individuals was associated with declines in global cognitive functioning, abstract reasoning and verbal memory [6]. Taken together, current evidence suggests that even modest declines in kidney function 
should be of concern in people with late Stage 2-4 chronic kidney disease.

Although our understanding of the process of cognitive aging remains limited, this study highlighted the possibility that routinely assessed measures for estimating renal function may help to identify a population at greater risk of cognitive decline. A number of possible explanations for these findings exist. First, this relationship may be due to the accumulation of uremic toxins, some of which are accumulated in excess and sometimes even with mild renal impairment. Second, the brain and the kidney both have low vascular resistance and may be more susceptible to age-related fluctuations in flow leading to microvascular damage prior to the onset of large vessel disease [7]. Lastly, this relationship may be due to shared risk factors such as impaired glycemic control [8] or chronic inflammation [9]. Support for the first explanation can be derived from studies of cognitive function pre- and post-kidney transplantation that have reported improvements in cognitive function in patients after transplantation [10]. Irrespective of the exact mechanism(s), the findings by Seliger and colleagues [2] once again highlight the importance of early detection of chronic kidney disease.

However, like other studies in this area, this study was observational and as such could not establish whether early chronic kidney disease was causative, interacts with other factors or is merely the harbinger of early vascular brain injury. Until intervention trials have been performed, these questions will remain unanswered. In this context, a number of strengths and limitations of the study by Seliger and colleagues [2] must be considered. The strengths included the detailed assessment of known risk factors with multiple longitudinal measures of renal function and cognitive performance. Limitations of the study included the use of creatinine as the sole determinant of renal function and the lack of assessment of structural brain changes over time.

In conclusion, the study by Seliger and colleagues [2] in this issue should stimulate further research investigating the link between declining renal function and cognition. The study also highlights the importance of early detection of chronic kidney disease to identify people at risk of cognitive impairment and provides a strong evidence that the process may begin much earlier than first thought. As such, studies are needed to demonstrate that interventions preventing the progression of early kidney disease can also reduce the burden of cognitive aging.

\section{Conflict of Interest}

Dr. Lewis has no conflicts of interest to declare.

\section{References}

1 Blazer D, Yaffe K, Liverman C (eds); Institute of Medicine: Cognitive Aging: Progress in Understanding and Opportunities for Action. Washington, DC, The National Academies Press, 2015.

2 Seliger SL, Wendell CR, Waldstein SR, Ferrucci $\mathrm{L}$, Zonderman $\mathrm{AB}$ : Renal function and long-term decline in neurocognitive function: the Baltimore longitudinal study of aging. Am J Nephrol 2015;41:305-312.

3 Sajjad I, Grodstein F, Kang JH, Curhan GC, Lin J: Kidney dysfunction and cognitive decline in women. Clin J Am Soc Nephrol 2012; 7:437-443.

4 Kurella Tamura M, Wadley V, Yaffe K, McClure LA, Howard G, Go R, Allman RM, Warnock DG, McClellan W: Kidney function and cognitive impairment in US adults: the reasons for geographic and racial differences in stroke (REGARDS) study. Am J Kidney Dis 2008;52:227-234.

5 Kurella Tamura M, Muntner P, Wadley V, Cushman M, Zakai NA, Bradbury BD, Kissela B, Unverzagt F, Howard G, Warnock D, McClellan W: Albuminuria, kidney function, and the incidence of cognitive impairment among adults in the United States. Am J Kidney Dis 2011;58:756-763.

6 Davey A, Elias MF, Robbins MA, Seliger SL, Dore GA: Decline in renal functioning is associated with longitudinal decline in global cognitive functioning, abstract reasoning and verbal memory. Nephrol Dial Transplant 2013;28:1810-1819.

7 O’Rourke MF, Safar ME: Relationship between aortic stiffening and microvascular disease in brain and kidney: cause and logic of therapy. Hypertension 2005;46:200-204.
8 Ravona-Springer R, Moshier E, Schmeidler J, Godbold J, Akrivos J, Rapp M, Grossman HT, Wysocki M, Silverman JM, Haroutunian V, Beeri MS: Changes in glycemic control are associated with changes in cognition in non-diabetic elderly. J Alzheimers Dis 2012;30:299309.

9 Teunissen CE, van Boxtel MP, Bosma $\mathrm{H}$, Bosmans E, Delanghe J, De Bruijn C, Wauters A, Maes M, Jolles J, Steinbusch HW, de Vente J: Inflammation markers in relation to cognition in a healthy aging population. J Neuroimmunol 2003;134:142150.

10 Kaya Y, Ozturkeri OA, Benli US, Colak T: Evaluation of the cognitive functions in patients with chronic renal failure before and after renal transplantation. Acta Neurol Belg 2013;113:147-155. 